\title{
A Novel Energy Efficient Hierarchical Multi Level Cluster Routing Protocol based on LEACH
}

\author{
Greeshma Arya \\ Department of Electronics \\ Engineering \\ Indira Gandhi Delhi Technical \\ University \\ Delhi, India
}

\author{
D S Chauhan \\ Department of Electrical \\ Engineering \\ Uttarakhand Technical University \\ Dehradun, \\ India
}

\author{
Annu Shokeen \\ Department of Electronics \\ Engineering \\ Indira Gandhi Delhi Technical \\ University \\ Delhi, India
}

\begin{abstract}
As the use of Wireless sensor networks (WSNs) has grown enormously in the past few decades, the need of scalable \& energy efficient routing and data aggregation protocol for large scale deployment has also risen. LEACH is a hierarchical clustering protocol that provides an elegant solution for such protocols. One deficiency that affects the performance of the protocol is existence of very large and very small clusters in the network at the same time. This leads to decrease in lifetime of WSNs. Sensors operate on battery of limited power, so it is a great challenging aim to design an energy efficient routing protocol, which can minimize the delay while offering highenergy efficiency and long span of network lifetime. In this paper, a new protocol is proposed. The proposed protocol is energy efficient multi level cluster-based routing protocol for continuous stream queries in WSN for large network areas. We incorporate multi hop concept into LEACH [3] by dividing clusters into levels as in TL-LEACH [8] and consider a set of cluster heads, head-set, for cluster-based routing. The head-set members are responsible for control and management of the network. On rotation basis, a head-set member receives data from the neighboring nodes and from previous level, then aggregated data is transmitted to node of next level and so on, until it reaches the distant base station. For a given number of data collecting sensor nodes, the number of control and management nodes can be systematically adjusted to reduce the energy consumption, which increases network life and multi hop transmission reduces energy consumption as distance between transmitting nodes is reduced.
\end{abstract}

\section{Keywords}

Cluster based routing, Energy efficient, Multilevel, Head Set Members, Wireless sensor networks.

\section{INTRODUCTION}

A Wireless sensor network (WSN) refers to a group of spatially dispersed and dedicated sensors for monitoring and recording the physical conditions of the environment and organizing the collected data at a central location. WSNs measure environmental conditions like temperature, sound, pollution levels, humidity, wind speed and direction, pressure, etc. The power of wireless sensor networks lies in the ability to deploy large numbers of tiny nodes that assemble and configure themselves. Usage scenarios for these devices range from realtime tracking, to monitoring of environmental conditions, to ubiquitous computing, to monitoring of the health of organs. While often referred to as wireless sensor networks, they can also control actuators that extend control from cyberspace into the physical world. Recent advancements in IC and micro-electro- mechanical systems allow wireless sensor network to be constructed with numerous low power consuming, inexpensive micro sensors. By networking large numbers of tiny sensor nodes, it is possible to obtain data about physical phenomenon that is difficult or impossible to obtain in more conventional ways. As the cost of manufacturing sensor nodes continues to drop, an increase in deployments of WSNs is expected in coming years.

Since energy is the major constrain in WSNs, therefore to solve the energy constraints problem of WSNs various energy efficient protocols have already been proposed such as LEACH [3], APTEEN, HEED, PAMAS. The main objective of routing protocols is wireless sensor networks are to find approach for improvement of energy efficient and reliable transmission of sensed data to the base station. In recent years much research work on power aware routing has been done.

\section{RELATED WORK}

Heinzelman et al. [3] describes the LEACH protocol, which is a hierarchical self-organized cluster-based approach for monitoring applications. The data collection area is randomly divided into several clusters. Based on time division multiple access (TDMA), the sensor nodes transmit data to the cluster heads, which aggregate and transmit the data to the base station. A new set of cluster heads are chosen after specific time intervals. A node can be re-elected only when all the remaining candidates have been elected.

Deng Zhixiang and Qi Bensheng [8] propose Three Layered LEACH (TL-LEACH) which divides clusters into levels. Each cluster head are elected during set up phase of LEACH. They don't communicate directly to base station. Another set of cluster heads is selected to send data to the base station which is second level. This reduces the nodes which communicate directly to the base station thus saving unnecessary power dissipation hence the lifetime of the network is prolonged.

The proposed protocol incorporates the idea of three-layered LEACH with concept of head-set for the control and management of every cluster. This protocol is suitable particularly for large area networks for continuous monitoring of numerous widespread sensors, which are at a large distance from the base station. Further energy dissipation model is considered for more analysis.

There are two types of transmission techniques for large wireless sensor networks: 


\subsection{Direct Transmission}

In this technique all sensor nodes transmit their data directly to BS. This is extremely expensive in terms of energy consumed, since the BS may be very far away from some nodes. For example as shown in figure 1 energy consumed to transmit data to base station will be more because energy is directly proportional to square of the distance.

$$
\begin{aligned}
& E_{\text {transmit_direct }} \propto\left(3 d_{1}+d_{2}\right)^{2} \\
& E_{\text {transmit_direct }} \propto 9 d_{1}^{2}+d_{2}^{2}+6 d_{1} d_{2}
\end{aligned}
$$

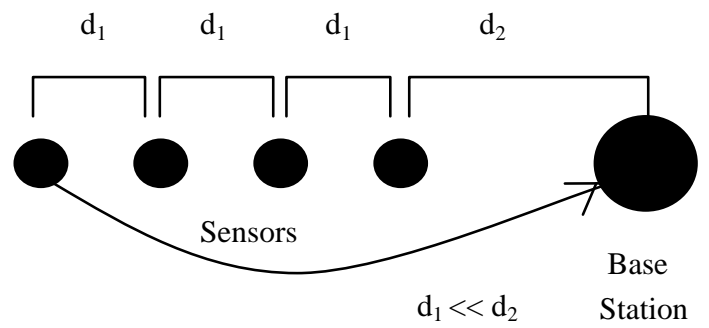

Figure 1 Direct Transmission

\subsection{Multi-hop Transmission}

In multi hop transmission, data is routed through the neighboring nodes in order to travel minimum distance to the base station via hopping technique as shown in figure 2 .

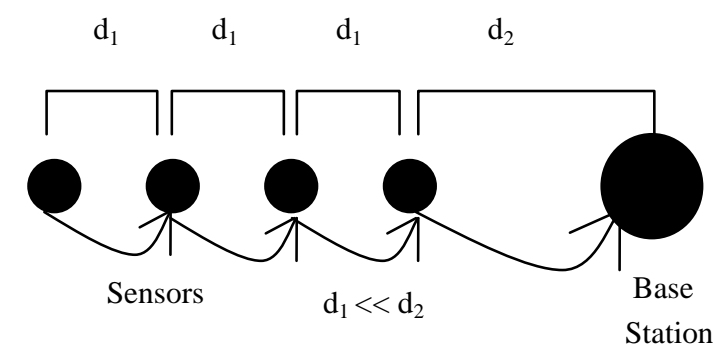

Figure 2 Multi-hop transmission

The energy consumed to transmit data to base station will be less,

$E_{\text {transmit_multi hop }} \propto\left(d_{1}^{2}+d_{1}^{2}+d_{1}^{2}+d_{2}^{2}\right)$

$E_{\text {transmit_multi hop }} \propto 3 d_{1}^{2}+d_{2}^{2}$

Since it is seen $E_{\text {transmit_multihop is much less than }}$ $E_{\text {transmit_direct }}$, hence energy consumption is reduced in multihop transmission.

\section{LEACH PROTOCOL}

Low-Energy Adaptive Clustering Hierarchy [3] is one of the most popular cluster-based routing protocols in wireless sensor networks. The operation of LEACH is broken up into rounds, where each round begins with a set-up phase. In each set up phase the clusters are formed followed by a steady-state phase where data transfer to the base station occurs. In order to minimize overhead, the steady-state phase is long compared to the set-up phase. To reduce management consumption, the steady-state phase is much longer compared to the set-up phase.

\subsection{Set-up Phase}

In the set-up phase, initially the node becomes a cluster-head with a probability $\mathrm{P}$ and broadcasts its decision packet. The regular nodes choose their cluster-head based on the least communication energy to reach the cluster-head. The role of the cluster-head keeps on rotating among the nodes of the cluster to enhance the network life time. The selection of cluster-head depends on decision made by the node by generating a random number between 0 and 1 . If the number is less than a threshold $\mathrm{T}(\mathrm{n})$, the node becomes a cluster-head for the current round. The threshold is set as:

$$
T(n)=\left\{\begin{array}{cc}
\frac{\mathrm{P}}{1-\mathrm{P} *\left(\operatorname{rmod}^{1} / p\right)}, & \text { if } n \in G \\
0 & \text { otherwise }
\end{array}\right.
$$

Where $\mathrm{P}$ equals the suggested percentage of cluster-heads, $\mathrm{r}$ is the current round, and $G$ is the set of nodes that have not been cluster-heads in the last $1 / \mathrm{P}$ rounds. By using this threshold, each node will be a cluster-head at some point within 1/ $\mathrm{P}$ rounds. During the first round $(\mathrm{r}=0)$, each node has a probability $\mathrm{P}$ of becoming a cluster-head. The nodes that are cluster-heads in round 0 cannot be cluster-heads for the next $1 / \mathrm{P}$ rounds. Thus the probability that the remaining nodes are clusterheads must be increased, since there are fewer nodes that are eligible to become cluster-heads. After $1 / \mathrm{P}-1$ rounds, $\mathrm{T}=1$ for any nodes that have not yet been cluster-heads, and after $1 / \mathrm{P}$ rounds, all nodes are once again eligible to become cluster-heads.

Once the cluster-heads have been chosen, the cluster-heads use CSMA MAC protocol to broadcast advertisement messages to the rest of the nodes. The regular nodes must keep their receivers on during this phase to hear the advertisements of all the clusterheads. After this phase, each regular node decides which cluster to join for the current round. Then the regular node will inform the cluster-head that it will become a member of the cluster. Each regular node transmits this information back to the cluster-head again using a CSMA MAC protocol. The cluster-head receives all the messages for nodes that would like to join in the cluster. Based on the number of regular nodes in the cluster, the clusterhead creates a TDMA schedule telling each regular node when it can transmit. This schedule is broadcast back to the regular nodes in the cluster. This is the whole process of the set-up phase.

\subsection{Steady-state Phase}

After the clusters are created and the TDMA schedule is fixed, data transmission can begin. The regular node will send data during their allocated transmission time to the cluster-head according to the TDMA schedule. The radio of each regular node can be turned off until the node's allocated transmission time. The cluster-head will keep its receiver on to receive all the data from the nodes in the cluster. When all the data has been received, the cluster-head performs data fusion functions to compress all the data into a single signal. After that the composite signal is sent to the base station directly by the clusterhead. Since the base station is far away, this is a high energy transmission. This is the steady-state operation of LEACH networks. After a certain time, which is determined a priori, the next round begins. 


\section{PROPOSED PROTOCOL}

Energy efficient hierarchical multi level cluster based routing protocol is an extension based on LEACH [3]. It is designed to be implemented in a WSN that has a large network diameter, about three times as that of leach which being a single hop protocol limits the diameter of the WSN. In contrast, it is a multihop protocol. The sensor nodes in a WSN that implements the proposed protocol are grouped into clusters using cluster formation algorithm. These clusters are divided into different levels i.e. L1,L2,L3 based on their distances from the base station, with the closest clusters from the BS being the highest level as shown in figure 3. Cluster formation algorithm is similar to LEACH and we extend cluster head selection algorithm by using a head-set instead of a cluster head. In other words, during each election, a head-set that consists of several nodes is selected. The members of a head-set are responsible for transmitting messages to the distant base station. At one time, only one member of the head-set is active and the remaining head-set members are in sleep mode. The task of transmission to the base station is uniformly distributed among all the head-set members.

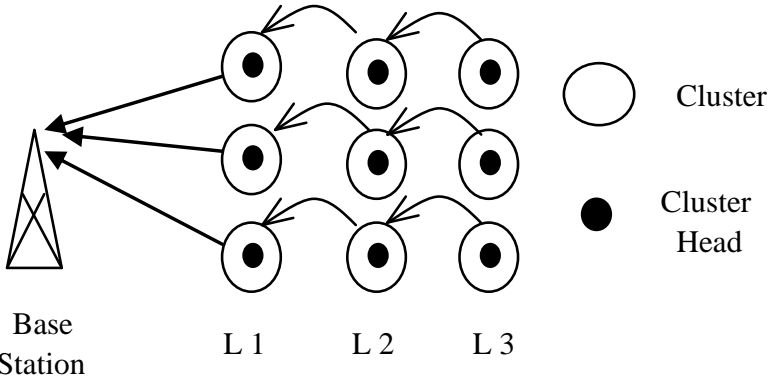

\section{Figure 3 Energy efficient hierarchical multi level cluster based routing protocol}

A cluster-head is a sensor node that transmits an aggregated sensor data to the distant base station. Non-cluster heads are sensor nodes that transmit the collected data to their cluster head. Each cluster has a head-set that consists of several virtual cluster heads; however, only one head-set member is active at one time. Each iteration consists of two stages: an election phase and a data transfer phase. In an election phase, the head-sets are chosen for the pre-determined number of clusters. In the data transfer phase, the members of head-set transmit aggregated data to the base station. Each data transfer phase consists of several epochs. Each member of a head-set becomes a cluster head once during an epoch. A round consists of several iterations. In one round, each sensor node becomes a member of head-set for one time. The above communication stages are illustrated in figure 4 .

\begin{tabular}{|c|c|c|c|c|}
\hline \multirow[t]{4}{*}{ Round } & \multirow[t]{2}{*}{ Iteration } & \multirow{2}{*}{$\begin{array}{l}\text { Election } \\
\text { phase }\end{array}$} & Epoch 1 & \multirow{2}{*}{$\begin{array}{l}\text { Data } \\
\text { transfer }\end{array}$} \\
\hline & & & Epoch $\mathrm{j}$ & \\
\hline & \multirow{2}{*}{$\begin{array}{l}\text { Iteratio } \\
n\end{array}$} & \multirow{2}{*}{$\begin{array}{l}\text { Electio } \\
\mathrm{n} \text { nhase }\end{array}$} & Epoch & \multirow{2}{*}{$\begin{array}{l}\text { Data } \\
\text { transfer }\end{array}$} \\
\hline & & & Epoch $\mathrm{j}$ & \\
\hline \multirow[t]{2}{*}{ Round $?$} & \multirow[t]{2}{*}{ Iteration } & \multirow{2}{*}{$\begin{array}{l}\text { Election } \\
\text { phase }\end{array}$} & Epach_1_ & \multirow{2}{*}{\} $\begin{array}{l}\text { Data } \\
\text { transfer }\end{array}$} \\
\hline & & & Epoch $j$ & \\
\hline
\end{tabular}

Figure 4 Communication stages in a cluster of a wireless sensor network.

\subsection{States of a Sensor Node}

Different states of a sensor node in a wireless sensor network are shown in figure 5. The damaged or malfunctioning sensor states are not considered. Each sensor node joins the network as a candidate. At the start of each iteration, a fixed number of sensor nodes are chosen as cluster heads; these chosen cluster heads acquire the active state. By the end of election phase, a few nodes are selected as members of the head-sets; these nodes acquire associate state. At the end of an election phase, one member of a head-set is in active state and the remaining head-set members are in associate state. In an epoch of a data transfer stage, the active sensor node transmits a frame to the base station and goes into the passive associate state. Moreover, the associate, which is the next in the schedule to transmit to the base station, acquires the active state. During an epoch, the head-set members are distributed as follows: one member is in active state, a few members are in associate state, and a few members are in passive associate state. During the transmission of the last frame of an epoch, one member is active and the remaining members are passive associates; there is no member in an associate state. Then, at the start of the next epoch, all the head-set members become associate members and one of them is chosen to acquire the active state.

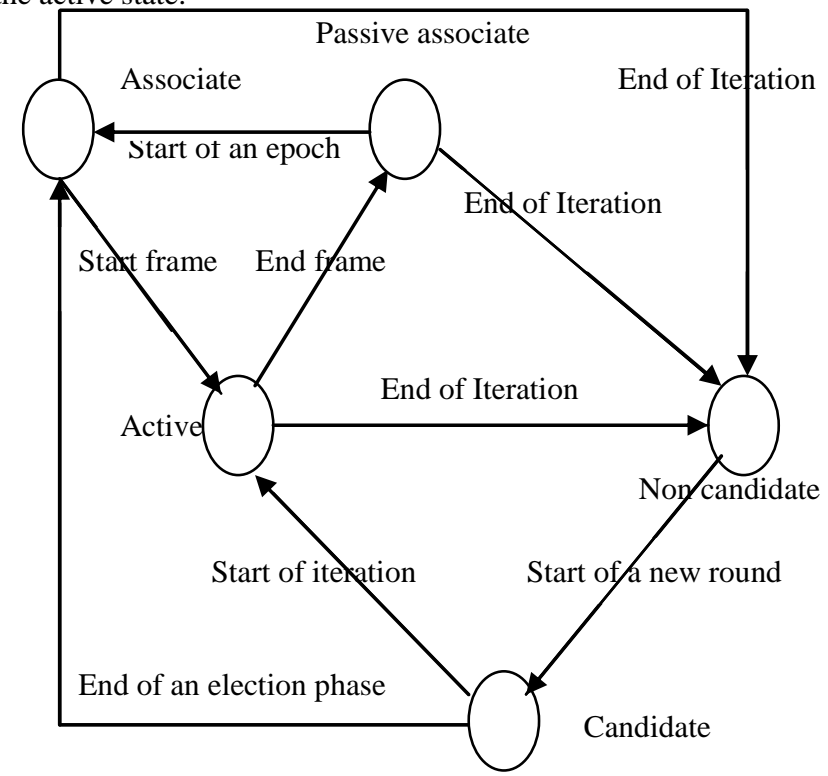

Figure 5 States of a sensor node in a wireless sensor network.

At the end of iteration, all the head-set members acquire the noncandidate state. The members in non-candidate state are not eligible to become a member of a head-set. At the start of a new round, all non-candidate sensor nodes acquire candidate state; a new round starts when all the nodes acquire non-candidate state.

\subsection{Cluster Head Selection Algorithm}

The proposed protocol inherits the self configuring and randomized cluster formation algorithms from LEACH despite an increase in network area. Cluster heads at each level are elected simultaneously using LEACH's CH selection algorithm. One key difference is that in the proposed protocol an upperlevel $\mathrm{CH}$ is eligible to become a super-cluster-head $(\mathrm{SCH})$ for the lower level CHs. In this case, the lower-level $\mathrm{CHs}$ transmit aggregated data to an upper-level SCH instead to the BS. In other words, data collected at lower levels are relayed back to the BS through one or more upper-level SCHs. 


\subsection{Election Phase}

In the proposed model, the number of clusters, $\mathrm{k}$, are predetermined for the wireless sensor network. At the start, a set of cluster heads are chosen on random basis. These cluster heads send a short range advertisement broadcast message. The sensor nodes receive the advertisements and choose their cluster heads based on the signal strengths of the advertisement messages. Each sensor node sends an acknowledgment message to its cluster head. Moreover, for each iteration, the cluster heads choose a set of associates based on the signal analysis of the acknowledgments. A head-set consists of a cluster head and the associates. The head-set, which is responsible to send messages to the base station, is chosen for an iteration of round. In an epoch of iteration, each member of the headset becomes a cluster head. All the head-set members share the same time slot to transmit their frames. Based on uniform rotation, a schedule is created for the head-set members for their frame transmissions; only the active cluster head transmits a frame to the base station. Moreover, a schedule is created for the data acquisition and data transfer time intervals for the sensor nodes that are not members of the head-set.

\subsection{Data Transfer Phase}

Once clusters, head-sets, and TDMA-based schedules are formed, data transmission begins. The non-cluster head nodes collect the sensor data and transmit the data to the cluster head, in their allotted time slots. The cluster-head node must keep its radio turned on to receive the data from the nodes in the cluster The associate members of the head-set remain in the sleep mode and do not receive any messages. After, some pre-determined time interval, the next associate becomes a cluster head and the current cluster head becomes a passive head-set member.

At the end of an epoch, all the head-set members have become a cluster head for once. There can be several epochs in iteration. At the end of iteration, the head-set members become non-candidate members and a new head-set is chosen for the next iteration. Finally, at the end of a round, all the nodes have become noncandidate members. At this stage, a new round is started and all the nodes become candidate members.

To send all the collected data back to the BS efficiently, the data transfer phase operation of proposed protocol starts at the lowest level. At the beginning, local data is collected simultaneous at all levels. Each sensor node takes turn at transmitting data to their $\mathrm{CH}$. The aggregated data is then sent to an upper-level $\mathrm{SCH}$ or the BS. The difference from the LEACH operation is that in proposed protocol, an upper level $\mathrm{SCH}$ does not begin transmitting to a higher level SCH until it has received all the aggregated data coming from the lower level. After it receives all the data coming from the lower level, it sends both the data from the lower level and the same level to another upper-level SCH or to the BS, depending on how many levels exist in the WSN. At the end of the round, data collected at leach level is sent to the BS by level $1 \mathrm{SCHs}$.

\begin{tabular}{|l|l|}
\hline $\begin{array}{l}\text { Lowest Level CH } \\
\text { to higher level SCH } \\
\text { transmission }\end{array}$ & \begin{tabular}{|l|} 
Highest level \\
SCH to BS \\
transmission
\end{tabular} \\
\hline------ Frame ----- & \multicolumn{1}{|c|}{ Time } \\
\hline
\end{tabular}

\section{Figure 6 Data transfer operation}

There are a couple of observations regarding the steady-state phase of proposed protocol. First, an upper-level SCH is most likely unable to perform data aggregation on data received from lower level because this data is highly unlikely to be correlated to the data collected at its same level since it is collected by nodes very far away. As a result, whatever is received from the lowerlevel is accumulated into the overall data to be sent. Secondly, as the levels increase in a WSN, sensor nodes closer to the BS are burdened with more bits to transmit. They are more susceptible to running out of power. Furthermore, if the data collected faraway form the BS is to be transmitted back to the BS, then it must be transmitted in a relay fashion. In the proposed protocol, the $\mathrm{CHs}$ rotate among all nodes at each level, so the energyintensive tasks such as long range transmissions are distributed among all nodes.

\section{QUANTITATIVE ANALYSIS}

We use a radio communication model as described in [3] in the quantitative analysis of the proposed protocol. The radio model is defined as follows:

The energy consumed to transmit an $l$-bit message for a shorter distance $d$ is given by:

$E_{T}=l * E_{e}+E_{S} d^{2}$

(1)

The energy consumed to transmit an $l$-bit message for a longer distance $d$ is given by:

$E_{T}=l * E_{e}+E_{l} d^{4}$

(2)

Moreover, the energy consumed to receive the $l$-bit message is given by:

$E_{R}=l * E_{e}$

(3)

Parameters used in (1), (2) and (3) are described in Table 1. 
Table 1 Constants and parameters used in MATLAB simulations

\begin{tabular}{|c|c|c|}
\hline Description & Symbol & Value \\
\hline $\begin{array}{c}\text { Energy consumed by the amplifier to } \\
\text { transmit at a shorter distance }\end{array}$ & $E_{S}$ & $\begin{array}{c}10 \\
\mathrm{pJ} / \mathrm{bit} / \mathrm{m}^{2}\end{array}$ \\
\hline $\begin{array}{c}\text { Energy consumed by the amplifier to } \\
\text { transmit at a longer distance }\end{array}$ & $E_{l}$ & $\begin{array}{c}0.0013 \\
\mathrm{pJ} / \mathrm{bit} / \mathrm{m}^{4}\end{array}$ \\
\hline $\begin{array}{c}\text { Energy consumed in the electronics } \\
\text { circuit to transmit or receive the } \\
\text { signal }\end{array}$ & $E_{e}$ & $50 \mathrm{~nJ} / \mathrm{bit}$ \\
\hline $\begin{array}{c}\text { Energy consumed for data } \\
\text { aggregation. }\end{array}$ & $E_{D A}$ & $5 \mathrm{~nJ} / \mathrm{bit}$ \\
\hline $\begin{array}{c}\text { Number of bits in a message } \\
\text { Number of nodes in the network }\end{array}$ & $\mathrm{n}$ & 4000 \\
\hline Number of clusters in the network & $\mathrm{k}$ & $5 \mathrm{to} 60$ \\
\hline Number of headsets in the network & $\mathrm{m}$ & 1,3 \\
\hline Network Diameter & $\mathrm{M}$ & $\begin{array}{c}100 \mathrm{~m} \text { to } \\
550 \mathrm{~m}\end{array}$ \\
\hline Number of Levels in the network & $\mathrm{L}$ & 2,3 \\
\hline
\end{tabular}

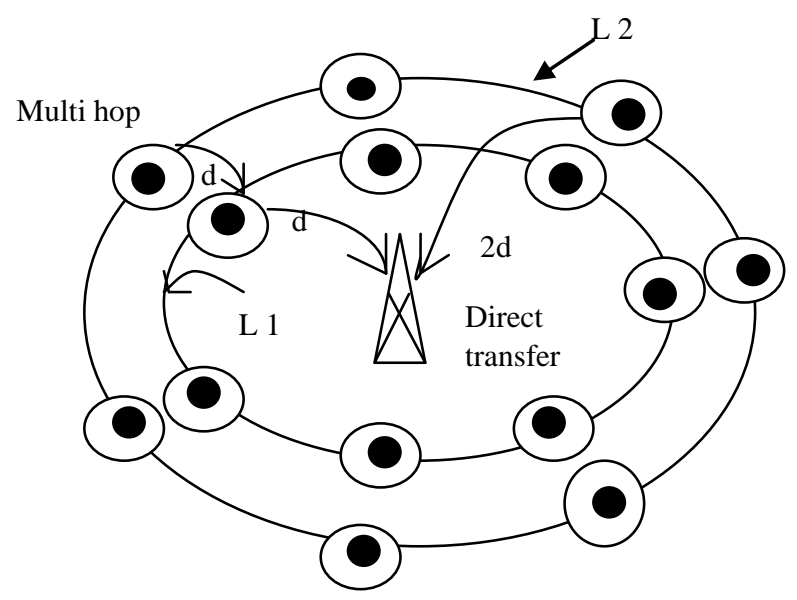

Figure 7

The proposed protocol is suitable particularly for large area networks for continuous monitoring of numerous widespread sensors, which are at a large distance from the base station. Therefore we have considered large area of network for our protocol and having large set of sensor nodes (assuming the network diameter to be $300 \mathrm{~m}-500 \mathrm{~m}$.). Initially two levels are considered and assumed that network is uniform i.e. every cluster in level 2 i.e. L2 transmits its data to the very next cluster of level 1 i.e. L1 and all clusters are in uniform sequence. Distances between clusters of level 1 and level 2 is assumed to be $d$ and same $d$ distance is considered between BS and levell clusters as shown in figure 7. Total number of nodes in the network is $n$ and $k$ is the total number of clusters. Therefore, there are $\frac{n}{k}$ nodes in every cluster. As we have considered network to be uniform, hence there are $\frac{k}{2}$ clusters in both levels. Total number of head set members is considered to be $m$. Therefore, there are $\frac{n}{k}-m$ number of nonCH nodes in a cluster.

\subsection{Total Energy of the Network}

Total energy of the network is the total energy consumption in the network for transferring data to BS. It is calculated for LEACH and the proposed protocol. The division of network into level is hypothetical to show the comparison between direct and multi hop transmission on the basis of transmission distance.

\subsubsection{LEACH}

There are two types of nodes in the network: head set members and non cluster head nodes. One of the head set member is cluster head i.e. in active mode and rest of them are in sleep mode. Since $\frac{n}{k}$ is the number of nodes in a cluster and $m$ is the number of head set members, and then $\frac{n}{k}-m$ are the non cluster head nodes. The energy consumed to transmit aggregated reading to Base Station for one cluster is considered to be the addition of energy consumed by cluster head and energy consumed by non cluster head in transferring data. Since there is one cluster head and $\frac{n}{k}-m$ non cluster head nodes, then energy consumed at level 2 by 1 cluster is:-

$E_{\text {level } 2 / \text { cluster }}=E_{\text {level } 2 \mathrm{CH}}+\left(\frac{n}{k}-m\right) E_{\text {level } 2 \text { nonCH }}$

(4)

$E_{\text {level } 2 \mathrm{CH}}$ is the energy consumed by cluster head for transferring data to the BS and $E_{\text {level } 2 \text { noncH }}$ is the energy consumed by non cluster head node for transferring data to the cluster head.

$E_{\text {level } 2 \text { nonCH }}=l * E_{e}+l * E_{s} * d^{2}$

(5)

For circular clusters with a uniform distribution of sensor nodes and a network diameter of $\mathrm{M}$, average value of $\mathrm{d}^{2}$ as in [9] is given by:

$$
d^{2}=\frac{M^{2}}{2 * \Pi * k}
$$

Since, we are considering large area networks, let us take network diameter to be twice of network considered for LEACH.. Therefore, the average value of $d^{2}$ will become $\frac{M^{2}}{\Pi * k}$. Therefore equation (5) is modified as:-

$E_{\text {level } 2 \text { nonCH }}=l * E_{e}+l * E_{s} *\left(\frac{M^{2}}{\Pi * k}\right)$

(6)

Equation (6) is used in both cases for calculating total network energy and start energy per node

$$
\begin{aligned}
E_{\text {level } 2 C H}= & \left(\frac{n}{k}-m\right) * l * E_{e}+l * E_{e}+l * E_{l} *(2 * d)^{4}+ \\
& \left(\frac{n}{k}-m+1\right) * l * E_{D A}
\end{aligned}
$$

(7)

Since distance is $2 * d$ between clusters of level 2 and BS as shown in figure 7 , its fourth power gives $16^{*} d^{4}$ which shows that large amount of energy is consumed in direct transmission for far away clusters. The third part of equation (7) represents energy consumed in data aggregation of $\left(\frac{n}{k}-m+1\right)$ frames of length $l$ - 
bit. To get the total energy of level 2, equation (4) is multiplied by $\frac{k}{2}$.

Similar procedure is adopted for level 1 to calculate energy consumed,

$\begin{aligned} E_{\text {level } 1 C H}= & \left(\frac{n}{k}-m\right) * l * E_{e}+l * E_{e}+l * E_{l} *(d)^{4}+\left(\frac{n}{k}-\right. \\ m+1) * l * E_{D A} & \end{aligned}$

Energy consumed by non cluster head is same as in (6) and total energy at level 1 is calculated by multiplying equation (4) by $\frac{k}{2}$. Total network energy is calculated by summation of energy consumed by both levels.

\subsubsection{Proposed Protocol}

As above mentioned that network has been divided into 2 levels, so we have derived equations for both levels. Cluster head of level 1 act as super cluster head for level 2 . First the cluster head of level 2 transmits data to cluster head of level 1 and then cluster head of level 1 transmits level 2 data along with its own data to BS. Similar procedure as described above is adopted for calculations. Energy consumed by cluster head in level 2 is given by:-

$E_{\text {level } 2 C H}=\left(\frac{n}{k}-m\right) l E_{e}+l E_{e}+l E_{l} d^{4}+\left(\frac{n}{k}-m+1\right) l E_{D A}$ (9)

Similar procedure is adopted for level 1 . Since cluster head of level 1 is transmitting 2 frames, one for level 2 and second for its own members, therefore energy consumed by cluster head is:

$$
\begin{aligned}
& E_{\text {level } 1 C H}=\left(\frac{n}{k}-m+1\right) l E_{e}+2 * l E_{e}+2 * l E_{l} d^{4}+\left(\frac{n}{k}-\right. \\
& m+ \\
& 1) * l * E_{D A}
\end{aligned}
$$$$
\text { (10) }
$$

Energy consumed by non cluster head is given by (6) and is used for both levels. Total energy of each level is calculated by multiplying (4) by $\frac{k}{2}$. Total network energy is addition of energy required for both levels. Total energy for both protocols is simulated in MATLAB and about $80.06 \%$ of reduction in total energy is recorded in proposed.

\subsection{Start Energy for One Round}

\subsubsection{Election Phase}

For a sensor network of $n$ nodes, the optimal number of clusters is given as $k$. All nodes are assumed to be at the same energy level at the beginning. The amount of consumed energy is same for all the clusters. At the start of the election phase, the base station randomly selects a given number of cluster heads. First, the cluster heads broadcast messages to all the sensors in their neighborhood. Second, the sensors receive messages from one or more cluster heads and choose their cluster head using the received signal strength. Third, the sensors transmit their decision to their corresponding cluster heads. Fourth, the cluster heads receive messages from their sensor nodes and remember their corresponding nodes. For each cluster, the corresponding cluster head chooses a set of $m$ associates, based on signal analysis.

For uniformly distributed clusters, each cluster contains $\frac{n}{k}$ nodes. Equations for election phase will be same for both cases. Using
Equation (6) and Equation (3), the energy consumed by a cluster head is estimated as follows:

$E_{C H \_e l e c}=l * E_{e}+l * E_{S} *\left(\frac{M^{2}}{\Pi * k}\right)+\left(\frac{n}{k}-1\right) l * E_{e}$

The first part of Equation (11) represents the energy consumed to transmit the advertisement message; this energy consumption is based on shorter distance energy dissipation model. The second part of Equation (11) represents the energy consumed to receive $\frac{n}{k}-1$ messages from the sensor nodes of the same cluster. The energy consumed by non-cluster head sensor nodes is estimated as follows:

$E_{\text {nonCH_elec }}=l * E_{e}+l * E_{S} *\left(\frac{M^{2}}{\Pi * k}\right)+k * l * E_{e}$

The first part of equation (12) shows the energy consumed to receive messages from $k$ cluster heads; it is assumed that a sensor node receives messages from all the cluster heads. The second part of Equation (12) shows the energy consumed to transmit the decision to the corresponding cluster head.

\subsubsection{LEACH}

Election phase will remain same as explained in 5.2.1.

\subsubsection{Data Transfer Phase}

The energy consumed by a cluster head and non cluster head per frame at level 2 will be same as equation (6) and (7). In one iteration, $N_{f}$ data frames are transmitted. The frames transmitted by each cluster are $\frac{N_{f}}{k}$. The $\frac{N_{f}}{k}$ frames are uniformly divided among $\frac{n}{k}$ nodes of the cluster. Each cluster head frame transmission needs $\frac{n}{k}-m$ non-cluster head frames. For simplification of equations, the fractions $f_{1}$ and $f_{2}$ are given as below:

$$
\begin{aligned}
& f_{1} \\
& =\frac{1}{\frac{n}{k}-m+1}
\end{aligned}
$$

$f_{2}$

$=\frac{\frac{n}{k}-m}{\frac{n}{k}-m+1}$

The energy consumptions in a data transfer stage of each cluster are as follows:

$$
\begin{aligned}
& E_{\text {level2CH_data }}=f_{1} * \frac{N_{f}}{k} \\
& *\left(\frac{E_{\text {level } 2 \mathrm{CH}}}{\text { frame }}\right) \\
& E_{\text {level2nonCH_data }}=\frac{f_{2} * N_{f}}{k} *\left(E_{\frac{\text { level } 2 \text { nonCH H }}{\text { frame }}}\right)
\end{aligned}
$$

As there are $k$ clusters and $n$ nodes, in iteration, $m$ nodes are elected for every cluster. Thus, in each iteration $k^{*} m$ nodes are elected as members of head-sets. The number of iterations required for all $n$ nodes to be elected is $\frac{n}{\mathrm{~km}}$, which is the number of iterations required in one round. Moreover, iteration consists of an election phase and a data transfer stage. The energy 
consumed in iteration by cluster and non cluster head is summation of election phase and data transfer phase.

Since there $m$ nodes in a head-set, the $E_{\text {level 2_CH/iter/cluster }}$ i.e. energy consumed at level 2 by $\mathrm{CH}$ per iteration per cluster, is uniformly divided among the head-set members, as given below:

$E_{\text {level } 2 \mathrm{CH} / \text { node }}$

$=\left(E_{\text {level 2_CH/iter } / \text { cluster }}\right) \frac{1}{m}$

Similarly, there are $\frac{n}{k}-m$ non-cluster head nodes in a cluster. The $E_{\text {level2_nonCH /iter/cluster }}$ i.e. energy consumed at level 2 by non $\mathrm{CH}$ per iteration per cluster, is uniformly distributed among all the non-cluster head members as follows:

$$
E_{\text {level 2nonCH } / \text { node }}=\left(E_{\text {level2_nonCH } / \text { iter } / \text { cluster }}\right) \frac{1}{\frac{n}{k}-m}
$$

These equations are major factor reducing energy consumption per node for cluster head as energy consumption by one cluster head is divided among $m$ cluster heads. Therefore, this is increasing life time of every node. The start energy, $E_{\text {level } 2 \text { start }}$, is an energy of a sensor node at the initial start time at level 2 . This energy should be sufficient for at least one round. In one round, a node becomes a member of head-set for one time and a non-cluster head for $\frac{n}{k m}-1$ times. Therefore,

$$
\begin{aligned}
E_{\text {level 2start }}= & E_{\text {level } 2 \mathrm{CH} / \text { node }} \\
& +\left(\frac{\mathrm{m}}{\mathrm{km}}-1\right) E_{\text {level } 2 \text { nonCH } / \text { node }}
\end{aligned}
$$

So average $E_{\text {start }}$ is calculated:-

$E_{\text {startavg }}=\frac{E_{\text {level 1sta rt }}+E_{\text {level 2start }}}{2}$

(19)

\subsubsection{Proposed Protocol}

Election phase will remain same as explained in 5.2.1.

\subsubsection{Data Transfer Phase}

The energy consumed by a non cluster head and cluster head per frame at level 2 will be same as equation (6) and (9) respectively. Similar procedure as mentioned above is adopted to calculate start energy per node at level 2. Equations (16) and (17) are used to get energy consumption per node by cluster head and non cluster head respectively.
There will be few differences at level 1 because $\mathrm{CH}$ of level 1 transmits two frames instead of 1. Equations (6) and (10) are used to calculate energy consumed by a non cluster head and cluster head respectively. The energy consumptions in a data transfer stage of each cluster are as follows:

$E_{\text {level1_CH_data }}=f_{3} * N_{f} * E_{\text {level1_CH } / \text { frame }}$

(20)

$E_{\text {level1_nonCH_data }}=f_{4} * N_{f} * E_{\text {level1_nonCH } / \text { frame }}$ (21)

Where the fractions $f_{3}$ and $f_{4}$ are taken for simplification of equations, given as below:

$$
\begin{aligned}
& f_{3} \\
& =\frac{2}{\frac{n}{k}-m+2} \\
& f_{4}=\frac{\frac{n}{k}-m}{\frac{n}{k}-m+2}
\end{aligned}
$$

\section{RESULTS AND DISCUSSION \\ 6.1 Total Energy of Network for 2 Levels Proposed Protocol}
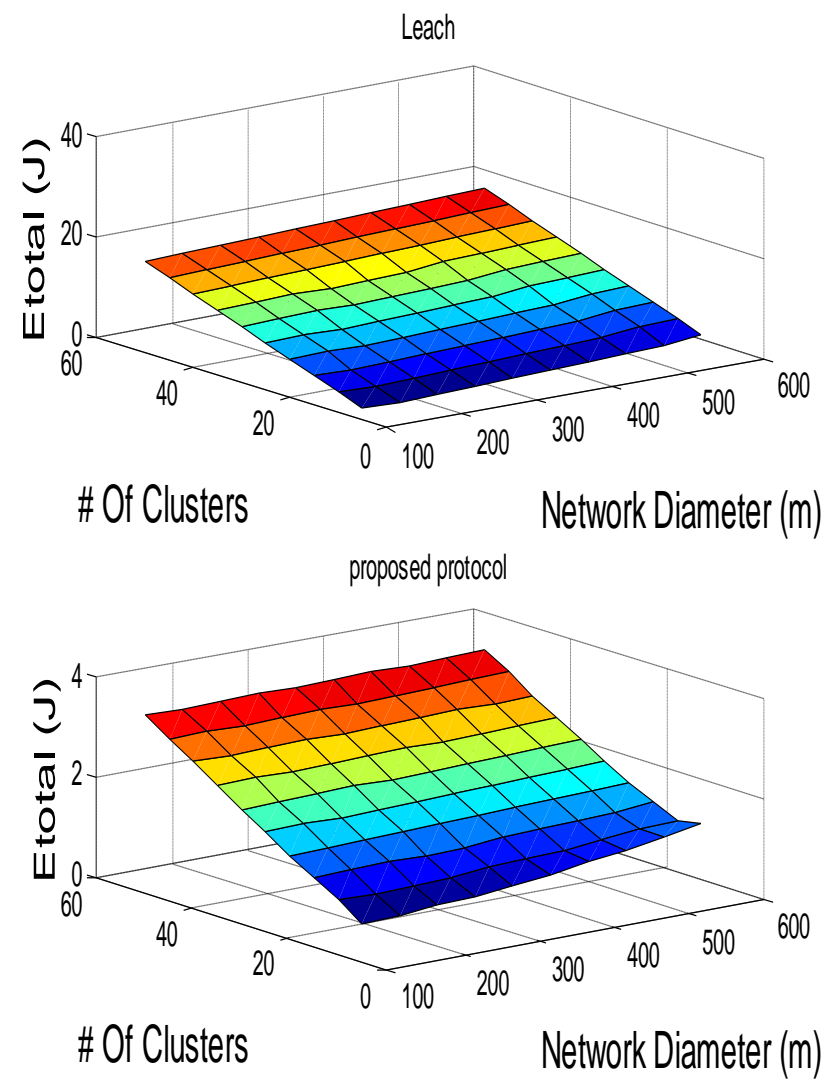

Figure 8 
Figure 8 compares total energy of network using LEACH protocol and proposed protocol. Same parameters are used for both i.e. $n=1000, d=300 \mathrm{~m}, \mathrm{l}=4000, \mathrm{M}=100 \mathrm{~m}, 150 \mathrm{~m}, 200 \mathrm{~m}$, $250 \mathrm{~m}, 300 \mathrm{~m}, 350 \mathrm{~m}, 400 \mathrm{~m}, 450 \mathrm{~m}, 500 \mathrm{~m}, 550 \mathrm{~m}$ and $k=5,10,15,20,25,30,35,40,45,50$. Proposed protocol is plotted for 2 levels and head set size of 3 . The $\mathrm{x}$-axis and $\mathrm{y}$-axis represent number of clusters and network diameter respectively. From MATLAB simulation we have analyzed that energy consumption for large network areas is reduced about $80.0653 \%$ as compared to LEACH for same large area. This concludes that proposed protocol is suitable for larger area network having network diameter about 300 to $500 \mathrm{~m}$ where if $\mathrm{LEACH}$ is used large energy is consumed because of increased size of network.

Figure 9 compares the total energy of network with respect to the levels in which we have divided it. An equation is derived through which we can get total energy of network for any number of levels and same equation is plotted here. The $\mathrm{x}$-axis and $y$-axis represent number of clusters and levels of network in which it is divided respectively.

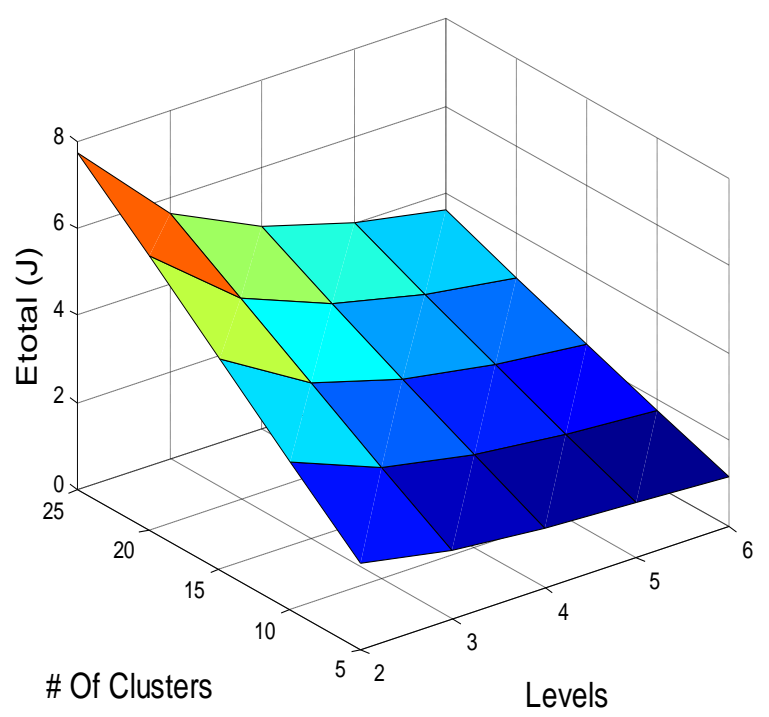

Figure 9

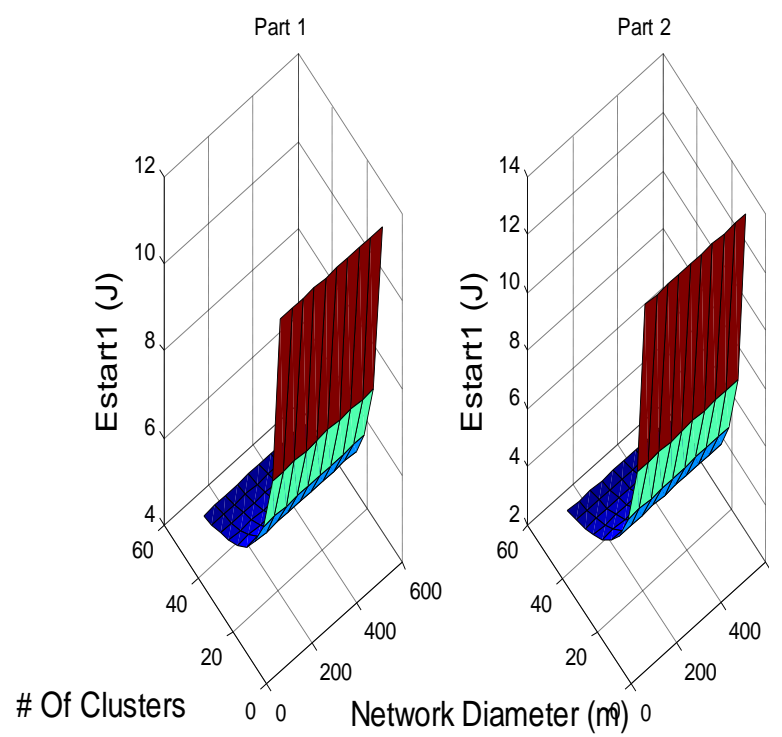

Figure 11
Parameters used are $\mathrm{m}=3, \mathrm{n}=1000, \mathrm{~d}=300 \mathrm{~m}, \mathrm{l}=4000, \mathrm{M}=100 \mathrm{~m}$, $150 \mathrm{~m}, 200 \mathrm{~m}, 250 \mathrm{~m}, 300 \mathrm{~m}, 350 \mathrm{~m}, 400 \mathrm{~m}, 450 \mathrm{~m}, 500 \mathrm{~m}, 550 \mathrm{~m}$. The graph shows that as we increase the number of levels for same size of network i.e. $M=200$, energy consumption reduces. We have analyzed from MATLAB simulation that as we increases levels from $\mathrm{L}=2$ to $\mathrm{L}=6$, about $53.39 \%$ energy consumption reduces of the total network.

Figure 10 shows comparison between total network energy for 2 levels and 3 levels for same head set size i.e. 3 and $n=1000$, $\mathrm{d}=300 \mathrm{~m}, \mathrm{l}=4000, \mathrm{M}=100 \mathrm{~m}, 150 \mathrm{~m}, 200 \mathrm{~m}, 250 \mathrm{~m}, 300 \mathrm{~m}, 350 \mathrm{~m}$, $400 \mathrm{~m}, 450 \mathrm{~m}, 500 \mathrm{~m}, 550 \mathrm{~m}$ and $\mathrm{k}=5,10,15,20,25,30,35,40,45,50$. The $\mathrm{x}$-axis and $\mathrm{y}$-axis represent number of clusters and network diameter respectively. We analyze from graph that $22.5616 \%$ energy consumption is reduced as we increase from 2 levels to 3 levels.

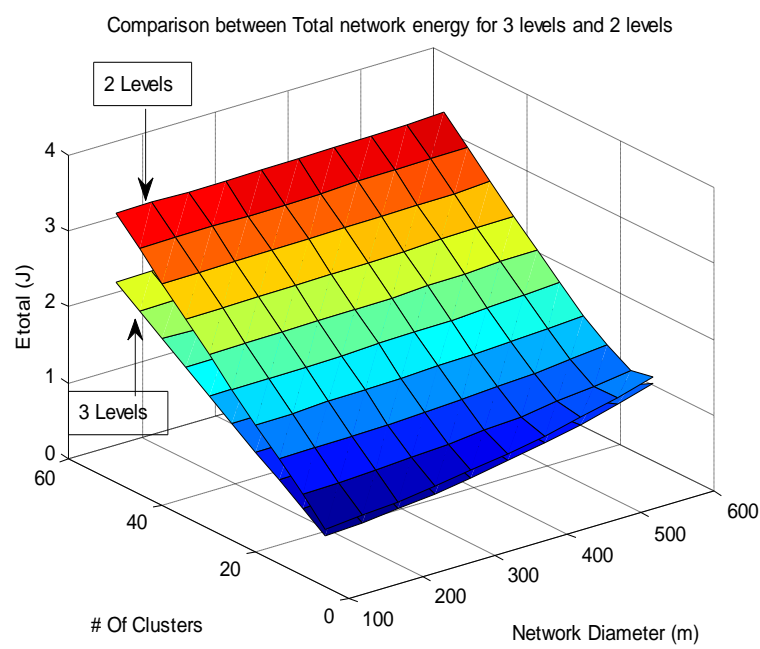

Figure 10 


\subsection{Start Energy per node in the Network}

Part 1 and Part 2 of figure 11 shows the energy consumption per node required initially for LEACH and proposed protocol with head set size $m=1$ respectively using same parameters i.e. $\mathrm{n}=1000, \mathrm{~d}=300$

$\mathrm{m}, \mathrm{L}=2, \mathrm{l}=4000, \mathrm{M}=100 \mathrm{~m}, 150 \mathrm{~m}, 200 \mathrm{~m}, 250 \mathrm{~m}, 300 \mathrm{~m}$, $350 \mathrm{~m}, \quad 400 \mathrm{~m}, 450 \mathrm{~m}, \quad 500 \mathrm{~m}, \quad 550 \mathrm{~m}$ and $\mathrm{k}=5,10,15,20,25,30,35,40,45,50$. The $\mathrm{x}$-axis and $\mathrm{y}$-axis represent number of clusters and network diameter respectively. Figures show that using proposed protocol with $m=1$ gives $13.6679 \%$ reduction in start energy per node. This shows that multi hop transmission reduces about $13.6679 \%$ of energy per node. Part 3 and part 4 of figure 11 compares start energy per node for direct transmission and multi hop transmission respectively with same head set size $m=3, L=2, n=1000, d=300 \mathrm{~m}$, $\mathrm{l}=4000, \mathrm{M}=100 \mathrm{~m}, 150 \mathrm{~m}, 200 \mathrm{~m}, 250 \mathrm{~m}, 300 \mathrm{~m}, 350 \mathrm{~m}$, $400 \mathrm{~m}, 450 \mathrm{~m}, 500 \mathrm{~m}, 550 \mathrm{~m}$ and $\mathrm{k}=5,10,15,20,25,30$, $35,40,45,50$. The $x$-axis and y-axis represent number of clusters and network diameter respectively. This justifies our incorporation of multi-hop transmission in protocol as about $15.2613 \%$ start energy per node is reduced. Part 2 and part 4 of figure 11 shows that on increasing head set size for proposed protocol from $m=1$ to $m=3$, the start energy per node reduces about $66.1237 \%$ for same parameters as discussed above. It justifies incorporation of head set instead of single cluster head because now the energy consumption by cluster head is distributed among all head set members. Therefore, reducing start energy per node and thereby increasing network life time. Part 1 and part 4 of figure 11 compares LEACH protocol and proposed protocol with $\mathrm{m}=3$ with respect to start energy per node. Same parameters are used as discussed above. The start energy reduces about $70.7539 \%$ when we use Energy efficient multi level cluster based routing protocol with $m=3$ and 2 levels.

\section{CONCLUSION AND FUTURE WORK}

The results of our quantitative analysis of the energy efficient multi level cluster based protocol indicate that the energy consumption of total network can be systematically decreased by increasing the no. of levels in the network. Also, energy consumption per node can be reduced by including more sensors in a head-set. For the same number of data collecting sensor nodes, the number of control and management nodes can be adjusted according to the network environment.
In future work, the variation in the head-set size for different network conditions will be investigated. This work will be extended to incorporate non-uniform cluster distributions. Data aggregation of un-correlated data will be worked upon to decrease start energy per node.

\section{REFERENCES}

[1] S. Bandyopadhyay and E. J. Coyle. An energy efficient hierarchical clustering algorithm for wireless sensor networks. In Proceedings of the IEEE Conference on Computer Communications (INFOCOM), 2003.

[2]http://en.wikipedia.org/wiki/Wireless_sensor_network

[3] W. R. Heinzelman, A. Chandrakasan, and H. Balakrishnan. Energy-efficient communication protocol for wireless microsensor networks. In Proceedings of the Hawaii International Conference on System Sciences, January 2000.

[4] Fan Xiangning, Song Yulin. Improvement on LEACH Protocol of Wireless Sensor Networks. In 007 International Conference on Sensor Technologies and Applications.

[5] Sun Limin, Li Jianzhong, Chen Yu, Wireless Sensor Networks.Tsinghua publishing company, Beijing, 2005.

[6] D. Estrin, "Tutorial Wireless Sensor Networks-Part IV: Sensor Network Protocols", MobiCom, 2002.

[7] S. Lindsey and C. S. Raghavendra. PEGASIS: Power-efficient gathering in sensor information systems. In Proceedings of the IEEE Aerospace Conference, March 2002.

[8] Deng Zhixiang, Qi Bensheng. Three-layered Routing Protocol for WSN Based on LEACH Algorithm. College of Computer \& Information Engineering, Hohai Univ., Changzhou 213022, China.

[9] Sajid Hussain and Abdul W.Matin. Energy efficient hierarchical cluster based routing for wireless sensor networks. Jodrey school of computer science, Acadia University. 